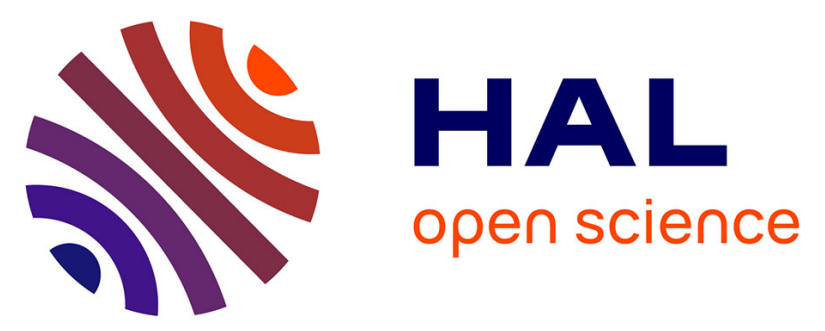

\title{
Assessing the biofuel production potential of Botryococcus braunii strains by sensitive rapid qualitative chemotyping using chemometrically-assisted gas chromatography-mass spectrometry
}

Benjamin Moutel, Marion André, Delphine Kucma, Jack Legrand, Dominique Grizeau, Jeremy Pruvost, Olivier Gonçalves

\section{To cite this version:}

Benjamin Moutel, Marion André, Delphine Kucma, Jack Legrand, Dominique Grizeau, et al.. Assessing the biofuel production potential of Botryococcus braunii strains by sensitive rapid qualitative chemotyping using chemometrically-assisted gas chromatography-mass spectrometry. Algal Research

- Biomass, Biofuels and Bioproducts, 2015, 11, pp.33 - 42. 10.1016/j.algal.2015.05.001 . hal-01887935

\section{HAL Id: hal-01887935 \\ https://hal.science/hal-01887935}

Submitted on 14 Apr 2020

HAL is a multi-disciplinary open access archive for the deposit and dissemination of scientific research documents, whether they are published or not. The documents may come from teaching and research institutions in France or abroad, or from public or private research centers.
L'archive ouverte pluridisciplinaire HAL, est destinée au dépôt et à la diffusion de documents scientifiques de niveau recherche, publiés ou non, émanant des établissements d'enseignement et de recherche français ou étrangers, des laboratoires publics ou privés. 


\title{
Assessing the biofuel production potential of Botryococcus braunii strains by sensitive rapid qualitative chemotyping using chemometrically-assisted gas chromatography-mass spectrometry
}

\author{
Benjamin Moutel ${ }^{\mathrm{a}}$, Marion André ${ }^{\mathrm{b}}$, Delphine Kucma ${ }^{\mathrm{a}}$, Jack Legrand ${ }^{\mathrm{a}}$, Dominique Grizeau ${ }^{\mathrm{a}}$, \\ Jérémy Pruvost ${ }^{\mathrm{a}}$, Olivier Gonçalves ${ }^{\mathrm{a}, *}$ \\ a LUNAM Université, Université de Nantes, CNRS, GEPEA, UMR 6144, Bât. CRTT, 37 Boulevard de l'Université, BP 406, F-44602 Saint-Nazaire Cedex, France \\ ${ }^{\mathrm{b}}$ Institut de Chimie des Substances Naturelles, CNRS UPR 2301, Avenue de la Terrasse, 91190 Gif-sur-Yvette, France
}

\begin{abstract}
A B S T R A C T
The microalga Botryococcus braunii has been studied for its hydrocarbon biofuel production potential. The range of the lipids it synthesizes cannot be readily profiled, because their main chemical class standards (long chain alkenes and botryococcenes) are at present unavailable.

The aim of this study was to develop a direct, specific GCMS analysis method for the rapid chemotyping of these lipids.

The SIM (Selected Ion Monitoring)-GCMS program we developed discriminates classes and subclasses of the targeted molecules through their main chemical motifs identified with fragment ions of specific $\mathrm{m} / \mathrm{z}$ selected by unsupervised chemometrics.

Our validation results indicate that the SIM mode enhanced their detection by significantly increasing the signalto-noise ratio ca. 20-fold. The profiling results from TL extracts of diverse B. braunii strains show that under the culture conditions tested, these strains could be distinguished by their chemical signatures, which also signaled different culture conditions involving various physiological states. We were able to make a rapid estimate of the biofuel potential of these strains, focusing on alkenes and botryococcenes.

The direct injection SIM-GCMS method was successfully applied here for rapid chemotaxonomy, displaying good sensitivity and specificity for new high molecular weight hydrocarbons from B. braunii.
\end{abstract}

\section{Introduction}

Algal biomass is currently one of the most widely studied bioresources in the search for the perfect sustainable biofuel alternative crop [24]. This interest is notably explained by the remarkable diversity of microalgae, which comprise some $200,000-800,000$ different species of which only 35,000 have been described [5]. The diversity of microalgae holds tremendous potential for novel biosynthesized chemical entities. Among the known oleaginous microalgae, the green colonial alga Botryococcus

Abbreviations: EI, electron impact; FA, fatty acid; (F)AME, (fatty) acid methyl ester; FC, fold change; FS, full scan mode; GC-FID, gas chromatography coupled to a flame ionization detector; GC-MS, gas chromatography coupled to mass spectrometry; HCs, hydrocarbons; $\mathrm{HC}$, hierarchical clustering; HCPC, hierarchical clustering on principal component analysis; ND, not detected; PCA, principal component analysis; TIC, total ion current; TLs, tota lipids; TL-HCs, total lipid hydrocarbons; TL-FAs, total lipid fatty acids; TG, triglyceride; NL, neutral lipid; PL, polar lipid; RSD, relative standard deviation; RT, retention time; SIM, selected ion monitoring; SPE, solid phase extraction; SNR, signal-to-noise ratio; WCA, whole cell analytic.

* Corresponding author.

E-mail address: olivier.goncalves@univ-nantes.fr (O. Gonçalves). braunii has been found to be of interest: under precise environmental conditions (natural or in a photobioreactor) it will accumulate large quantities of hydrocarbons instead of the commonly described triacylglycerol [26]. Based on the type of hydrocarbons they synthesized, B. braunii varieties were classified into three chemotypes, noted A, B and L. Chemotype A produced $n$-alkadienes and n-alkatrienes $\left(C_{25}-C_{31}\right)$, chemotype $B$ produced polymethylated unsaturated triterpenes called botryococcenes $\left(\mathrm{C}_{n} \mathrm{H}_{2 n-10}, n=30-37\right)$, and chemotype L produced a single tetraterpene named lycopadiene $\left(\mathrm{C}_{40} \mathrm{H}_{78}\right)$ [1]

The structural complexity of those molecules has been assessed and resolved mainly by Metzger and coworkers [14-22]. They developed lengthy preparative chromatographic approaches involving multiple purification steps to obtain analyzable fractions of extracellular or intracellular hydrocarbons. Short contact time with hexane first yielded extracellular hydrocarbons from lyophilized biomass [7], and then suitable protocols $[3,6]$ retrieved intracellular lipids. A combination of different solvent polarity applied directly on dried biomass was also tested by Kojima and Zhang [10], without using a preparative chromatographic approach. In both cases the amount of $B$. braunii biomass needed to perform the experiments was substantial, up to several 
grams of material. In most cases analysis was performed on very aged biomass known to accumulate larger amounts of hydrocarbons. The significantly long generation time of this microalga, i.e. 2-4 days, can be seen as an important limiting step for such studies.

Recent investigations making use of lipidomic-related techniques permitted the hydrocarbon phenotyping of two B. braunii races (A and B) using pyrolysis-gas chromatography-mass spectrometry [2]. Very small quantities of material were used ( $1 \mathrm{mg}$ of dried biomass), and no complex sample preparation was needed to achieve chemotyping of these races. The main chemical features of races A and B were recorded on the hydrocarbon pyrograms [8], with mass signatures associated with botryococcenes, nonacosadiene, eicosadiene, phytadiene, etc. However, the proposed structural assignment of the fragments obtained after mass spectrometry analysis relied on an in silico semi-automatic annotation pipeline and not on experimental fragmentation pattern data. This constraint is due to the absence of easily producible or commercially available standards for botryococcenes, alkadienes and trienes. Although such a chemotyping strategy was quite straightforward and parsimonious in terms of sample preparation, it implies destructive analysis, which caused numerous chemical rearrangements, generating chemical structures that could not always be easily elucidated [9]. The simpler sample preparation procedure was offset by additional difficulties in mass spectral data interpretation and structural resolution.

In the work of Mjøs [22], a very interesting strategy was highlighted for fatty acid structure prediction, especially applicable when reference standards were not available. The results of this study indicated that by means of chemometrics it was possible to solve the fatty acid methyl ester (FAME) structure of marine lipids from tiny differences in the relative abundance of all or some of the fragment ions characterized by their $m / z$ values in a low-resolution mass spectrum. By working in a very narrow mass range $(m / z=50-110)$ the author was able to assess structural differences such as numbers of double bonds, distinguishing polyenes from dienes, monoenes and saturated compounds. In addition, this approach revealed the existence of a subset of fragment ions suitable for selected ion monitoring (SIM) with predictivity close to that obtained in full scan mode. Adapting such a strategy for the structural characterization of the new lipids from $B$. braunii and especially its hydrocarbons had never been attempted before. It should surmount the absence of standards normally required for developing an assay method. Such an approach should make lipid analysis possible on an affordable low-resolution mass spectrometry system for chemical profiling, potentially enhanced for both identification and sensitivity. Concerning this last, the results of Mjøs [23] also suggest the possibility of remedying the signal loss typically observed for the hydrocarbon family by developing a very sensitive SIM mode [25].

In this work we explored the potential of such an approach, focusing on the development of a sensitive, easy-to-use qualitative analytical method for the hydrocarbon chemotyping of $B$. braunii races A and B. A gas chromatography-mass spectrometry profiling method was set up using chemometrics for optimized fragment ion selection and data interpretation. Our aim here was to propose an affordable analytical method that would make up for the lack of chemical standards and a sensitive technique for assessing targeted hydrocarbon diversity at early stages of the microalgal culture. Efforts were also made to use the biological material sparingly. The profiling method developed was applied in real conditions for the qualitative chemotyping of $B$. braunii races $A$ and $B$. Results and perspectives emerging from this study are described and discussed.

\section{Materials and methods}

\subsection{Chemicals and hydrocarbon standards}

Analytical grade solvents were purchased from VWR International. Butylated hydroxytoluene (BHT), boron trifluoride $\left(\mathrm{BF}_{3}, 14 \%\right.$ by weight in methanol), glyceryl triheptadecanoate (TAGs17:0), the 37 FAME components and tricosane were obtained from Sigma Aldrich.

Substitution standards were used for the development of the analytical methods, since the major hydrocarbons synthesized by B. braunii species were not commercially or academically available. Linear or branched alkadienes or trienes (race A), botryococcenes (race B), and lycopadiene (race $L$ ) were no longer available (Chiron $A S \circledR$, Trondheim, Norway). The n-alkane standard mixture $\left(\mathrm{C}_{10}-\mathrm{C}_{40}\right.$, all even), squalane, squalene and phytol, were respectively chosen as substitutes for these compounds, because they contain the major structural elements found in the hydrocarbons synthesized by $B$. braunii (Table 1 ). A substitution standard mixture was made up of FAME concentration set at $1.6 \mu \mathrm{g} \cdot \mu \mathrm{L}^{-1}$, and all other compounds between 20 and $90 \mathrm{ng} \cdot \mu \mathrm{L}^{-1}$ (n-alkane all even: $20 \mathrm{ng} \cdot \mu \mathrm{L}^{-1}$, squalane: $50 \mathrm{ng} \cdot \mu \mathrm{L}^{-}$, squalene: $70 \mathrm{ng} \cdot \mu \mathrm{L}^{-1}$ and phytol $\left.90 \mathrm{ng} \cdot \mu \mathrm{L}^{-1}\right)$.

\subsection{Strains and culture conditions}

Ten strains of $B$. braunii were purchased from international algae banks: Algoban (Caen, France), CCA (Scotland), CCALA (Czech Republic), and SAG (Germany). Table 2 gives their features and the codes used in this work.

The algal cells were cultured in $250 \mathrm{~mL}$ conical flasks under constant stirring at $75 \mathrm{rpm}$, constant pH 7.5 (maintained using $4 \% \mathrm{CO}_{2}$-enriched air bubbled at $20 \mathrm{~mL} \cdot \mathrm{min}^{-1}$ ) and constant illumination at approximately $80 \mu \mathrm{mol} \mathrm{m} \mathrm{m}^{-1}$ at $25{ }^{\circ} \mathrm{C}$ using modified Chu 13 medium, which contains 3 times higher concentrations of inorganic macronutrients and trace metals than the original Chu 13 medium [11].

\subsection{Analytical methods used on the culture medium}

All analyses were performed in triplicate. Culture turbidity was measured spectrophotometrically at $750 \mathrm{~nm}$. The algal dry weight (DW) measurements were determined by filtration through a pre-dried, preweighed $0.2 \mu \mathrm{m}$ glass-fiber filter (Whatman GF/F) with demineralized water used as washing buffer. The filter was dried for $24 \mathrm{~h}$ at $105^{\circ} \mathrm{C}$, cooled in a desiccator and then re-weighed to within $0.01 \mathrm{mg}$ on an XA 105 analytical balance (Mettler Toledo). For each particle concentration, microalgal cells were counted using a hemocytometer (Malassez device) with an optical microscope (Zeiss).

\subsection{Development of the chemotyping method}

\subsubsection{B. braunii biomass harvesting protocol}

All the B. braunii cell cultures were run to obtain a final biomass concentration of $\sim 0.5 \mathrm{~g} \cdot \mathrm{L}^{-1}$, which corresponded to very early stages in our batch cultivation protocol, i.e. $\sim 2$ days for usual cultivation duration in the range of 7-10 days in our conditions. Approx. $50 \mathrm{~mL}$ samples of fresh culture were taken from each replicate flask, and the whole volume was frozen at $-80{ }^{\circ} \mathrm{C}$. The frozen cells in their culture media were then lyophilized for $12 \mathrm{~h}$ at $4{ }^{\circ} \mathrm{C}$ (RP2V, SGD Sérail, Argenteuil, France) and stored at $-20{ }^{\circ} \mathrm{C}$ before undergoing the total lipid (TL) extraction protocol. This biomass sampling protocol was chosen because it avoided any leakage of the targeted metabolites during the cell harvesting procedure. It thus ensured acquisition of a faithful image of the compounds present in the analyzed matrix.

\subsubsection{TL extraction protocol}

The TL extraction protocol was developed for lyophilized B. braunii biomass as follows. The glassware was systematically heated for $6 \mathrm{~h}$ at $450{ }^{\circ} \mathrm{C}$ to prevent organic pollution. Aliquots of $50 \mathrm{mg}$ of lyophilized biomass sample were placed in glass vials in triplicate, and assayed for TL extraction. This consisted in extracting all the TLs, i.e. including the targeted hydrocarbons, irrespective of their cellular localization, using a chloroform/methanol mixture $(2 / 1 \mathrm{v} / \mathrm{v})$ as the single extraction solvent. It was adapted from the whole cell analytic (WCA) approach developed by Meier et al. [13]. 
Table 1

Substitution standards used to develop analytical methods for Botryococcus braunii hydrocarbons.

\begin{tabular}{|c|c|c|c|}
\hline Race & Major hydrocarbons & $\begin{array}{l}\text { Commercial substitution } \\
\text { standard }\end{array}$ & Characteristic chemical group \\
\hline \multirow[b]{2}{*}{ A } & \multirow{2}{*}{$\begin{array}{l}\text { alkanes, alkadienes and alkatrienes even } \\
\text { number of carbon atoms from } \mathrm{C}_{23} \text { to } \mathrm{C}_{33} \\
\text { (linear or branched) }\end{array}$} & $\begin{array}{l}\text { n-alkane mix from } \mathrm{C}_{10} \text { to } \\
\mathrm{C}_{40} \text { all even }\end{array}$ & $\begin{array}{l}\text { Linear alkyl motif } \\
\mathrm{C}_{n} \mathrm{H}_{2 n+1}\end{array}$ \\
\hline & & Squalane $\left(\mathrm{C}_{30} \mathrm{H}_{62}\right)$ & 2-methylbutyl motif \\
\hline B & $\begin{array}{l}\text { Polymethylated unsaturated triterpenes } \\
\text { (botryococcenes, } \mathrm{C}_{n} \mathrm{H}_{2 n-10}, n=30-37 \text { ) }\end{array}$ & Squalene $\left(\mathrm{C}_{30} \mathrm{H}_{50}\right)$ & Dimethylallyl motif \\
\hline & Lycopadiene tetraterpene $\left(\mathrm{C}_{40} \mathrm{H}_{78}\right)$ & Phytol $\left(\mathrm{C}_{20} \mathrm{H}_{40} \mathrm{O}\right)$ & notif $\left(\mathrm{C}_{20} \mathrm{H}_{38}\right)$ \\
\hline $\mathrm{L}$ & 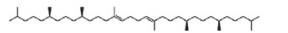 & & \\
\hline
\end{tabular}

One typical extraction sequence consisted in re-suspending the lyophilizate in about 4 volumes equivalent of ultrapure water, i.e. $40 \mu \mathrm{L}$, and then adding $2 \mathrm{~mL}$ of chloroform/methanol mixture $(2 / 1 \mathrm{v} / \mathrm{v})$. To ensure complete lipid extraction, the vials were sonicated in a water bath and shaken for $2 \mathrm{~h}$. To obtain maximum lipid depletion of the sample, the contact with the organic extracting mixture was repeated until no substances of interest could be detected in the pooled extracts (five cycles were assayed). Demixing was performed on the final $10 \mathrm{~mL}$ of organic extract by adding $2 \mathrm{~mL}$ of aqueous solution of $\mathrm{KCl}(0.8 \%)$ to remove the polar compounds from the TL solution. After decantation, the TL extract was evaporated to dryness and weighed to within $0.01 \mathrm{mg}$ for mass balance measurement (XA105DU analytical balance, Mettler Toledo); 0.01\% w/w of BHT (antioxidant) was then added to the dried total lipid (TL) extract, which was again evaporated to dryness, and finally stored at $-20{ }^{\circ} \mathrm{C}$ under nitrogen before chromatographic analysis.

\subsubsection{Fast semi-quantitative SIM-GCMS profiling of B. braunii lipids}

The TL extracts were analyzed directly, i.e. without pre-purification of the lipids on a SPE micro-column, on a GC-MS system for fast semiquantitative profiling. The analytical device used was a Trace GC Ultra (Thermo Fisher Scientific, Boston, MA, USA) coupled to a Trace ISQ single quadrupole mass spectrometer (Thermo Fisher Scientific, Boston, MA, USA). The optimized separation conditions for the measurement of the molecules of interest (HCs and FAMEs) were set up as follows: $2 \mu \mathrm{L}$ of sample was injected onto a TG-5HT column (apolar phase $5 \%$ phenyl methylpolysyloxane, internal diameter $30 \mathrm{~m} \times 250 \mu \mathrm{m}$, film thickness $0.25 \mu \mathrm{m}$; Thermo Scientifics, Waltham, MA, USA) using programmed temperature vaporization (PTV), injection set at constant temperature (CT) mode at $280{ }^{\circ} \mathrm{C}$, with a split mode of $1 / 10$ $\left(10 \mathrm{~mL} \cdot \mathrm{min}^{-1}\right)$. The flow rate of carrier gas (hydrogen produced by a generator; WM-H2, F-DGSi, Evry, France) during the analysis was $1.5 \mathrm{~mL} \cdot \mathrm{min}^{-1}$. The oven temperature gradient for the separation of the mixture of HCs plus FAMEs was $75{ }^{\circ} \mathrm{C}$ for 3 min, ramped at $10{ }^{\circ} \mathrm{C} \mathrm{min}{ }^{-1}$ to $300{ }^{\circ} \mathrm{C}$ for $1 \mathrm{~min}$, and finally at $100{ }^{\circ} \mathrm{C} \mathrm{min}-1$ to $340{ }^{\circ} \mathrm{C}$ for $4 \mathrm{~min}$ for cleaning. The temperature of the transfer line to the MS was $300{ }^{\circ} \mathrm{C}$, and the ion source was set at $320^{\circ} \mathrm{C}$. Electron ionization was conducted at $70 \mathrm{eV}$. For exploratory qualitative measurements (used for the development phase of the study and the assessment of the chemotypes of the 10 test strains), the MS was used in full scan (FS) mode throughout the mass range of 50-600 amu. For the improved sensitivity qualitative measurements (i.e. the chemotyping method specifically developed in this study), the MS was exploited using alternatively FS recording mode and selected ion monitoring (SIM) mode programmed with highly specific ions characteristic of the $B$. braunii lipids (see Table 4 in Section 3.4). These ions were selected using chemometric approaches included in the FactoMineR R package [12]. The mass resolution was 1 mass unit throughout the mass range of 50-600 amu. The total GC-MS analysis time was $30 \mathrm{~min}$. Data were post-processed and analyzed using the Xcalibur 2.1 software (Thermo Fisher Scientific, Waltham, MA, USA Thermo-Fisher).

\subsubsection{Selection of the ions specific to the new B. braunii lipids used to pro-} gram the SIM method

2.4.4.1. Theoretical mass spectral data extraction and organization of B. braunii lipids. Efficient SIM programming requires selecting the ion fragments that characterize and discriminate most of the classes of the molecules studied. For that purpose, the mass spectra of the compounds of interest were all retrieved from the NIST GC-MS database (version 2008) for the FAMEs (from C4:0 to C24:0) and the aliphatic linear hydrocarbons (HCs from C8 to C54). For the compounds characterizing the Botryococcus genus chemical signature, i.e. squalane, squalene, lycopadiene and botryals, mass spectral information was extracted from published data in the absence of commercially/academically available standards [17,18,20,21,23,24]. For the NIST 2008 data, a Perl (v. 5.10.0) script was developed for the parsing of the data in order to specifically extract for each molecule the 10 most abundant fragment ions and their corresponding relative abundances. Data were then organized into a table containing individuals (molecules) as columns, variables (fragment ions) as rows, and their relative abundance as informative data (normalized to 999). Table S1 summarizes the results obtained for the 75 selected compounds characterized by 72 fragment ions and their associated relative abundances.

2.4.4.2. Multivariate analysis of the mass spectral data. Principal component analysis (PCA) was performed on R 3.1.2 statistical software using the FactoMineR package [12]. Hierarchical clustering (HC) was systematically performed on the dataset to consolidate the study to yield a hierarchical clustering on principal component (HCPC) analysis. It empirically produced clusters of molecules based on their fragment ion signature differences, giving identification maps). Most significant (abundant) fragment ions were further selected through their calculated $v$ test and $p$ values to generate specific chemical signature classes; $m / z$

Table 2

Origin of the strains used.

\begin{tabular}{|c|c|c|c|c|c|}
\hline $\begin{array}{l}\text { Code used in this } \\
\text { work }\end{array}$ & $\begin{array}{l}\text { Original accession } \\
\text { number }\end{array}$ & Bank of origin & Race & Natural biotope & $\begin{array}{l}\text { References and reported } \\
\text { hydrocarbon amount (\%DW) }\end{array}$ \\
\hline bb1 & 755 & Algobank & A & Small pond (15, pH 6.9) Lingoult (France). & [17] (32\%) \\
\hline bb2 & 757 & Algobank & A & Small pond $\left(15^{\circ}, \mathrm{pH} 6.6\right)$ Oukaimeden (Morocco). & [17] (19 to $40 \%)$ \\
\hline bb3 & 761 & Algobank & $\mathrm{B}$ & Small pond ( $\left.32^{\circ}, \mathrm{pH} 6.6\right)$ Paquemar (Martinique). & [17] (28 to $38 \%)$ \\
\hline bb4 & 30.81 & SAG & A & Cuzco Lake (Peru) & [4] $(19 \%)$ \\
\hline bb5 & 777 & CCALA & - & - & - \\
\hline bb6 & 778 & CCALA & - & - & - \\
\hline bb7 & - & Confidential source & A & - & - \\
\hline bb8 & 835 & CCALA & A & Cuzco Lake (Peru) & [4] (19\%) \\
\hline bb9 & $807 / 2$ & CCAP & A & Grasmere (UK) & [10] (nd) \\
\hline bb10 & $807 / 1$ & CCAP & A & Cambridge (UK) & [11] (15\%) \\
\hline
\end{tabular}


values were selected when for an abundant candidate, both a positive $v$ test and very low $p$ value were observed (see [12] for details).

2.4.5. Validation of the fidelity of the separation conditions developed for the SIM-GCMS chemotyping method

To check that the separation on the apolar phase column of the SIMGCMS device of a non-pre-fractioned total lipid extract would preserve the detection of the chemical compounds of interest, namely the FAMEs and the HCS of B. braunii, we used SPE fractionation in a control experiment. Two kinds of fractionation were performed, one using the mixture of substitution standards for SPE calibration, the second using the crude TL extract for validation. GCFID was exploited as an independent analytical reference technique to assess, qualitatively and quantitatively, the fidelity of our chemical profiling approach. The experiments were also designed to compare the GCFID information with the SIM-GCMS semi-quantitative results.

2.4.5.1. SPE pre-purification approach. A $200 \mu \mathrm{L}$ aliquot of the $1 \mathrm{~mL}$ substitution standard mixture or TL extract was evaporated to dryness and resuspended in a total volume of $1 \mathrm{~mL}$ of amylene-stabilized chloroform $(1 \times 500 \mu \mathrm{L}$, followed by 2 volumes of $250 \mu \mathrm{L}$ with 30 s sonication and vortex agitation in between to optimize dissolution), and loaded onto a silica gel microcolumn (laboratory-made, length $70 \mathrm{~mm}$, i.d. $5 \mathrm{~mm}$, Kieselgel Merck 70-230 mesh silica gel previously heated at $450{ }^{\circ} \mathrm{C}$ and deactivated with $6 \%$ water by weight). Linear hydrocarbons, tricosane, and squalane (corresponding to the alkane, alkadiene and triene classes) were eluted with $8 \mathrm{~mL}$ of hexane, squalene (corresponding to the botryococcene class) with $8 \mathrm{~mL}$ of toluene, phytol (corresponding to the lycopadiene class) with $8 \mathrm{~mL}$ of dichloromethane, triglycerides (TG) with $8 \mathrm{~mL}$ of amylene-stabilized chloroform, neutral lipids different from the TG (LN-TG) with $8 \mathrm{~mL}$ of $\mathrm{CHCl}_{3}: \mathrm{MeOH}(98: 1)$, and the remaining polar lipids (LP) with $8 \mathrm{~mL}$ of methanol. The fractions were then stored at $-20{ }^{\circ} \mathrm{C}$ under nitrogen before analysis by GCFID.

2.4.5.2. Analysis of the organic fractions by the GCFID reference methods. The fractions from the SPE columns were redissolved in a chloroform/ methanol mixture $(2 / 1 \mathrm{v} / \mathrm{v})$ and aliquoted into two equal volumes prior to chromatographic analysis. One aliquot was used to analyze the quantity and quality of the total lipid hydrocarbons (TL-HCs) by specific gas chromatography coupled to a flame ionization detector (HCs GCFID). The second aliquot was used to analyze the quantity and quality of the total lipid fatty acids (TL-FAs) likewise by specific gas chromatography coupled to a flame ionization detector (FAMEs GCFID). For the two independent GCFID methods, the quantitative developments were achieved using both external and internal calibration curves per individual analytical standard with at least four data points (data not shown). The same procedure this time using our semi-quantitative GCMS profiling approach as an analytical technique was performed for comparison (on the same extracts as those used for the GCFID analysis). Table 3 details the analyses performed on the different SPE fractions.

2.4.5.3. Quantitative analysis of HCS by GCFID. The B. braunii total lipid (TL) fractions (substitution standards before SPE pre-purification, hexane fraction, toluene fraction and dichloromethane fraction) were used for the analysis of the quantity of the total lipid hydrocarbons (TL-HCs) by gas chromatography coupled to a flame ionization detector (GCFID). Analysis was performed on a $100 \mu \mathrm{L}$ aliquot of the TL extract to which $10 \mu \mathrm{g}$ of tricosane was added as an internal standard. HCs were analyzed with a 7820A gas chromatograph (Agilent Technologies, Santa-Clara, CA, USA) equipped with a split/splitless injector (injected quantity: $2 \mu \mathrm{L}$, split set at $1 / 10-10 \mathrm{~mL} \cdot \mathrm{min}^{-1}$ ), an HP-5 capillary column (apolar phase 5\% phenyl methylpolysiloxane, internal diameter $0.32 \mathrm{~mm}$, film thickness $0.25 \mu \mathrm{m}$, length $30 \mathrm{~m}$, Agilent Technologies, Santa-Clara, CA, USA) and a flame ionization detector. The flow rate of carrier gas (hydrogen) during the analysis was constant at $2.0 \mathrm{~mL} \cdot \mathrm{min}^{-1}$; the gas was produced using a hydrogen generator (WM-H2, F-DGSi,
Evry, France). The chromatograph temperature program was: injector temperature $280{ }^{\circ} \mathrm{C}$, FID $340{ }^{\circ} \mathrm{C}$, oven $75{ }^{\circ} \mathrm{C}$ for $3 \mathrm{~min}$, then ramped at $10^{\circ} \mathrm{C} \cdot \mathrm{min}^{-1}$ to $300^{\circ} \mathrm{C}$ for $15 \mathrm{~min}$. The HCs were identified by their retention times compared with known standard mixtures, i.e. the selected substitution standard commercial mixture described in Section 2.1. The quantities of HCs were determined from their respective peak areas relative to that of the tricosane internal standard. Integration and calculations were performed using the Agilent software Chemstation v. 0.1.0.4. The absolute quantities of the HCs were first expressed in $\mu$ g of TL-HCs in the analyzed sample, and in \%DW relative to the biomass concentration of the tested sample.

2.4.5.4. Quantitative analysis of FAMEs by GCFID. The B. braunii total lipid (TL) fractions (amylene-stabilized $\mathrm{CHCl}_{3}$ fraction, $\mathrm{CHCl}_{3}: \mathrm{MeOH}(98: 2)$ fraction, and $\mathrm{MeOH}$ fraction) were used to quantify the fatty acids (FAs) by gas chromatography coupled to a flame ionization detector (GCFID). The TL extracts were transesterified before the TL-FA GCFID analysis. The transesterification reaction was performed on a $100 \mu \mathrm{L}$ aliquot of the total lipid extract to which $10 \mu \mathrm{g}$ of TG17:0 was added as an internal standard. After evaporation to dryness under nitrogen and addition of $1 \mathrm{~mL}$ of $\mathrm{BF}_{3}$, the fractions were heated for $10 \mathrm{~min}$ at $100^{\circ} \mathrm{C}$. After cooling and adding $1 \mathrm{~mL}$ of hexane, the organic phase containing the fatty acid methyl esters (FAMEs) was washed three times with $1.5 \mathrm{~mL}$ of watersaturated hexane. FAMEs were then recovered and analyzed with a 7820A gas chromatograph (Agilent Technologies, Santa-Clara, CA, USA) equipped with a split/splitless injector (injected quantity $2 \mu \mathrm{L}$, split set at $1 / 10-10 \mathrm{~mL} / \mathrm{min}$ ), a TR-FAME capillary column (polar phase $70 \%$ cyanopropyl polysilphenylene-siloxane, internal diameter $0.25 \mathrm{~mm}$, thickness $0.25 \mu \mathrm{m}$ film, length $30 \mathrm{~m}$, Thermo Fisher Scientific, Waltham, MA, USA) and a flame ionization detector. The flow rate of carrier gas (hydrogen) during the analysis was constant at $1.0 \mathrm{~mL} \cdot \mathrm{min}^{-1}$; the gas was produced using a hydrogen generator (WM-H2, F-DGSi, Evry, France). The chromatograph temperature program was: injector temperature $250{ }^{\circ} \mathrm{C}$, FID $280{ }^{\circ} \mathrm{C}$, oven at $80{ }^{\circ} \mathrm{C}$ for $1 \mathrm{~min}$, then ramped at $5{ }^{\circ} \mathrm{C} \cdot \mathrm{min}^{-1}$ to $145^{\circ} \mathrm{C}$ for $12 \mathrm{~min}$, then at $1{ }^{\circ} \mathrm{C} \cdot \mathrm{min}^{-1}$ to $155^{\circ} \mathrm{C}$ for 5 min and finally at $5^{\circ} \mathrm{C} \cdot \mathrm{min}^{-1}$ to $200^{\circ} \mathrm{C}$ for $5 \mathrm{~min}$. The FAMEs were identified from their retention times compared with known standard mixtures such as the 37component FAME standard commercial mixture. The FAME quantities were determined from their respective peak areas relative to that of the TG17:0 internal standard. Integration and calculation were performed using the Agilent software Chemstation v. 0.1.0.4. The absolute quantities of the TL were first expressed in $\mu \mathrm{g}$ of TL-FAs in the analyzed sample and in \%DW relative to the biomass concentration of the test sample.

\subsubsection{Analysis of the chromatographic or total ionic current profiles}

Chemotyping data (TIC and chromatographic data) were analyzed semi-automatically (MS identification and coelution checking systematically underwent manual curation), and the final data matrix (retention index and relative or absolute abundance) was analyzed using the R 3.1.2 statistical software with the FactoMineR package [12]. PCA, HC and HCPC methods were used for data analysis and clustering.

Table 3

Analysis of the SPE fractions.

\begin{tabular}{lll}
\hline SPE fraction & Analysis method & \\
\cline { 2 - 3 } & Quantitative & Semi-quantitative \\
\hline No elution & HCs GCFID & FS or SIM-GCMS \\
Hexane & HCs GCFID & FS or SIM-GCMS \\
Toluene & HCs GCFID & FS or SIM-GCMS \\
Dichloromethane & HCs GCFID & FS or SIM-GCMS \\
Amylene-stabilized $\mathrm{CHCl}_{3}$ & FAMEs GCFID & FS or SIM-GCMS \\
$\mathrm{CHCl}_{3}:$ MeOH $(98: 2)$ & FAMEs GCFID & FS or SIM-GCMS \\
$\mathrm{MeOH}$ fraction & FAMEs GCFID & FS or SIM-GCMS \\
\hline
\end{tabular}




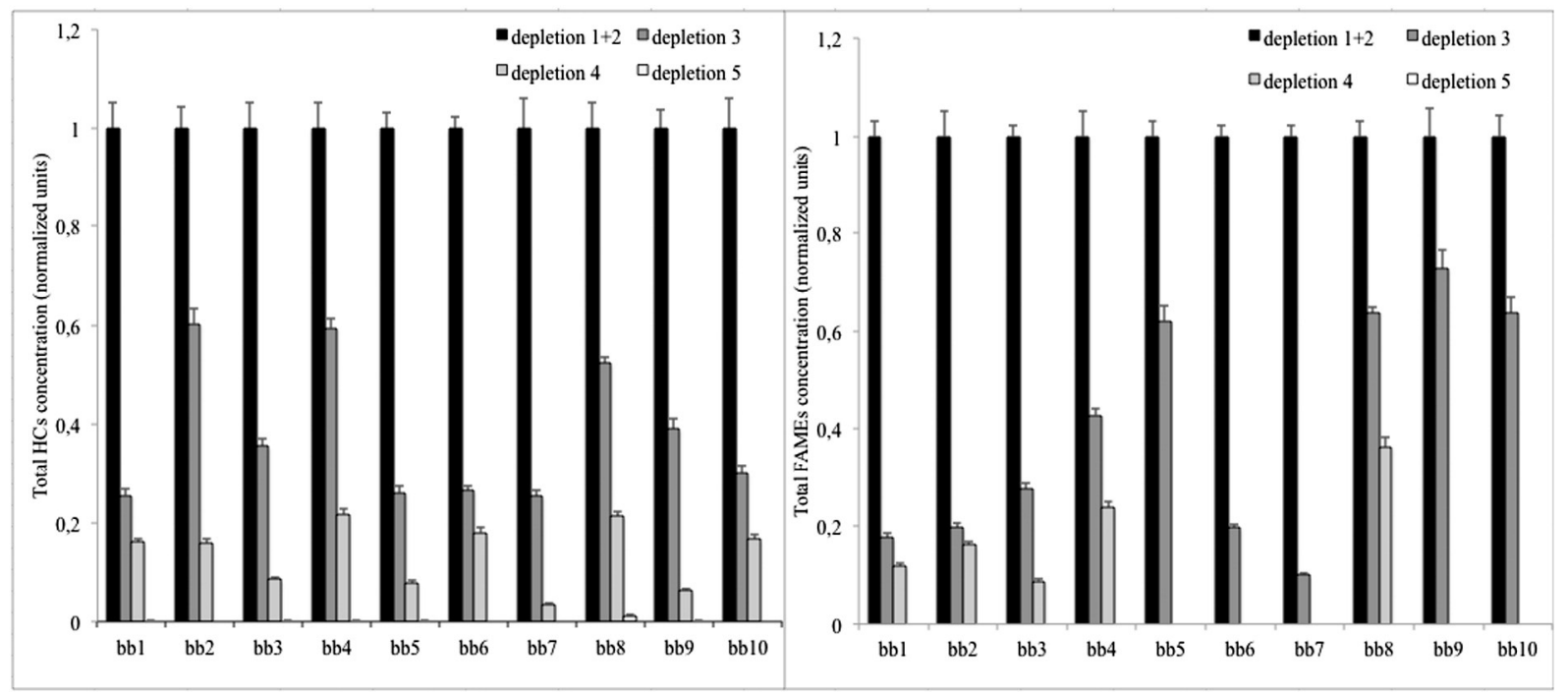

Fig. 1. Evolution of the normalized absolute content of HCs (left) and FAMEs (right) (\%DW) per Botryococcus braunii strain tested according to the number of successive extractions.

\section{Results and discussion}

In order to develop a simple, sensitive profiling method applicable directly on a non-pre-purified total lipid (TL) extract of Botryococcus cells, we focused on optimizing two major steps: first the extraction/ separation of the TL of interest and second the detection of these specific lipids. Hydrocarbons (linear and branched) and FAMEs were targeted and characterized as a priority, since they belong to the classes of

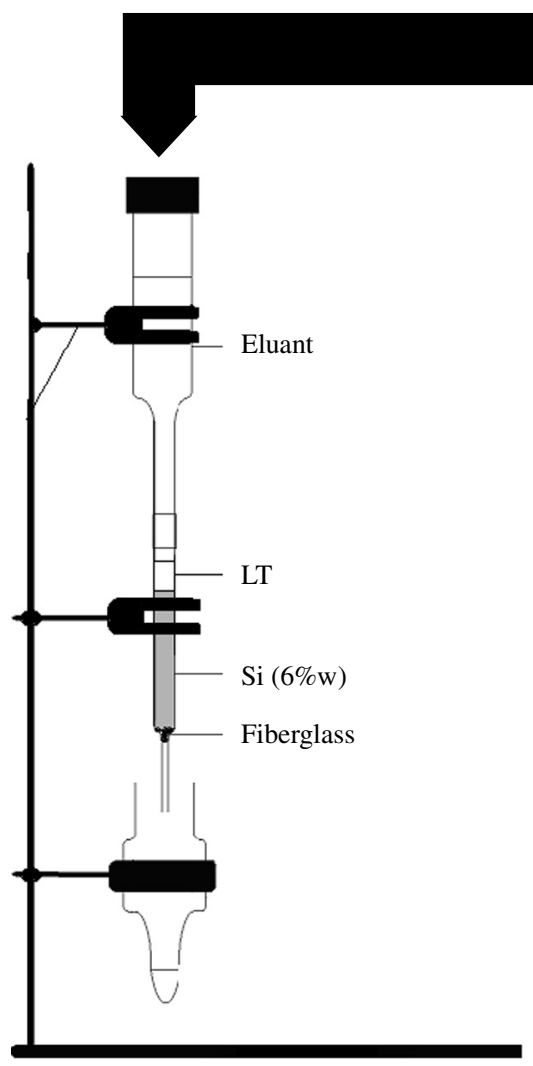

0 : substitution standards

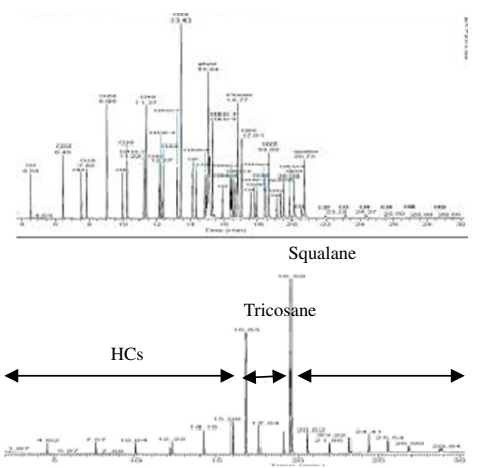

1: hexane fraction

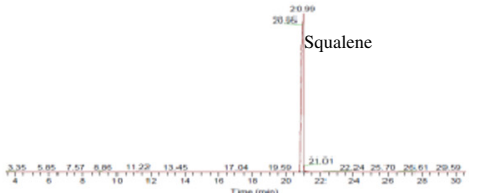

2: toluene fraction

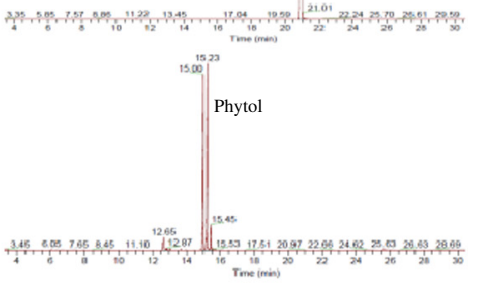

3: dichloromethane fraction

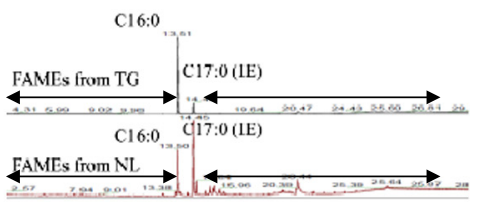

5: $\mathrm{CHCl}_{3}: \mathrm{MeOH}(98: 2)$

fraction

6: $\mathrm{MeOH}$ fraction

4: amylene-stabilized $\mathrm{CHCl}_{3}$ fraction

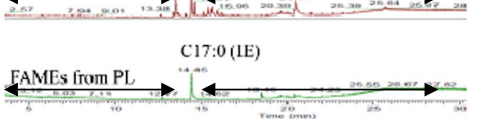

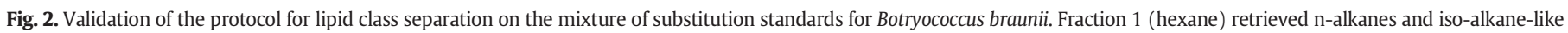
molecules (squalane), fraction 2 (toluene) squalene, fraction 3 (dichloromethane) phytol, and fractions 4,5 and 6 the FAMEs initially branched to the TG, the NL and the PL. 
substances biosynthesized by B. braunii races A and B, which are those being principally investigated for new biofuel alternatives (alkanes, botryococcenes and methylsqualenes).

\subsection{A general TL extraction protocol independent of the $10 \mathrm{~B}$. braunii strains tested was developed}

The extraction protocol using chloroform/methanol mixture $(2 / 1 \mathrm{v} / \mathrm{v})$ was chosen here because it allowed retrieval of the $B$. braunii TLs irrespective of their cellular location as previously described in the studies of Folch et al. [6]. The 10 strains (races A and B) were tested independently to assess the efficiency and repeatability of the extraction protocol. The strains underwent sequential extractions, and the extracts were analyzed using our two complementary reference methods for the analysis of FAMEs and HCs.

Concerning the efficiency of the extraction protocol, our results show that five extractions were needed to perform a quantitative depletion of the tested biomass without any detectable influence of the strain or race (Fig. 1). The repeatability of the protocol was also considered acceptable, the mean relative standard deviation (RSD) values calculated on the five independent replicates performed per strain being systematically less than $6 \%$ whatever the strain or race considered.

3.2. The separative conditions of the SIM-GCMS chemotyping method preserved the detection of the targeted lipids when directly analyzing the crude TL extract

In order to control the fidelity of the chemical information obtained in a single run with the SIM-GCMS chemotyping method, we performed control experiments using pre-purification of the lipid classes of interest on SPE columns. Comparison of the chromatograms of the crude extracts or substitution standards with those of the pre-purified HCs or of the other lipid classes showed that no significant differences could be measured with our method, whether qualitatively (Fig. 2), semiquantitatively or quantitatively. Concerning this last point, less than
$5 \%$ difference was found on comparing the quantification results of the GCFID analytical methods. This was considered non-significant, since it was less than the protocol intrinsic variability (data not shown). It shows that no loss of information was detectable when performing pre-purification of the targeted classes or using the crude extract or substitution standards in direct analysis mode with the SIM-GCMS chromatographic separation conditions (the same conclusion was drawn for the FS-GCMS method). This last finding is most important since it suggests that it is possible to avoid a lengthy protocol on a silica micro-column to separate the chemical classes of interest, and to simplify the analytical procedure by analyzing the TL crude extract directly.

3.3. However, the MS signal recorded for the substitution standards representative of the B. braunii lipids was found to be very heterogeneous and dependent on the chemical properties of the compounds

With the FS-GCMS conditions, the molecular ions of the linear aliphatic hydrocarbon standards were scarce, and their relative abundances fell exponentially as the number of carbon atoms increased (Fig. 3). The molecule's signal decreased when its number of carbon atoms exceeded $\mathrm{ca}$. 28. Similar observations were made for the FAME standards, with a decreasing signal observed for an increasing degree of unsaturation $(>1)$ and number of carbon atom $(n>16)$. This signal loss therefore needed compensation to maximize the chances of detecting the new molecules synthesized by $B$. braunii and for sensitive, nonbiased chemotyping.

3.4. An unsupervised chemometric approach was used to optimize the identification and detection of the main lipid classes of $B$. braunii according to their $m / z$ signature

To compensate for the loss of signal recorded for the profiled molecules in FS mode, a specific SIM mode using finely selected fragment ions was developed. The cluster analysis resulting from the HCPC approach performed on the entire dataset described in Section 2.4.4

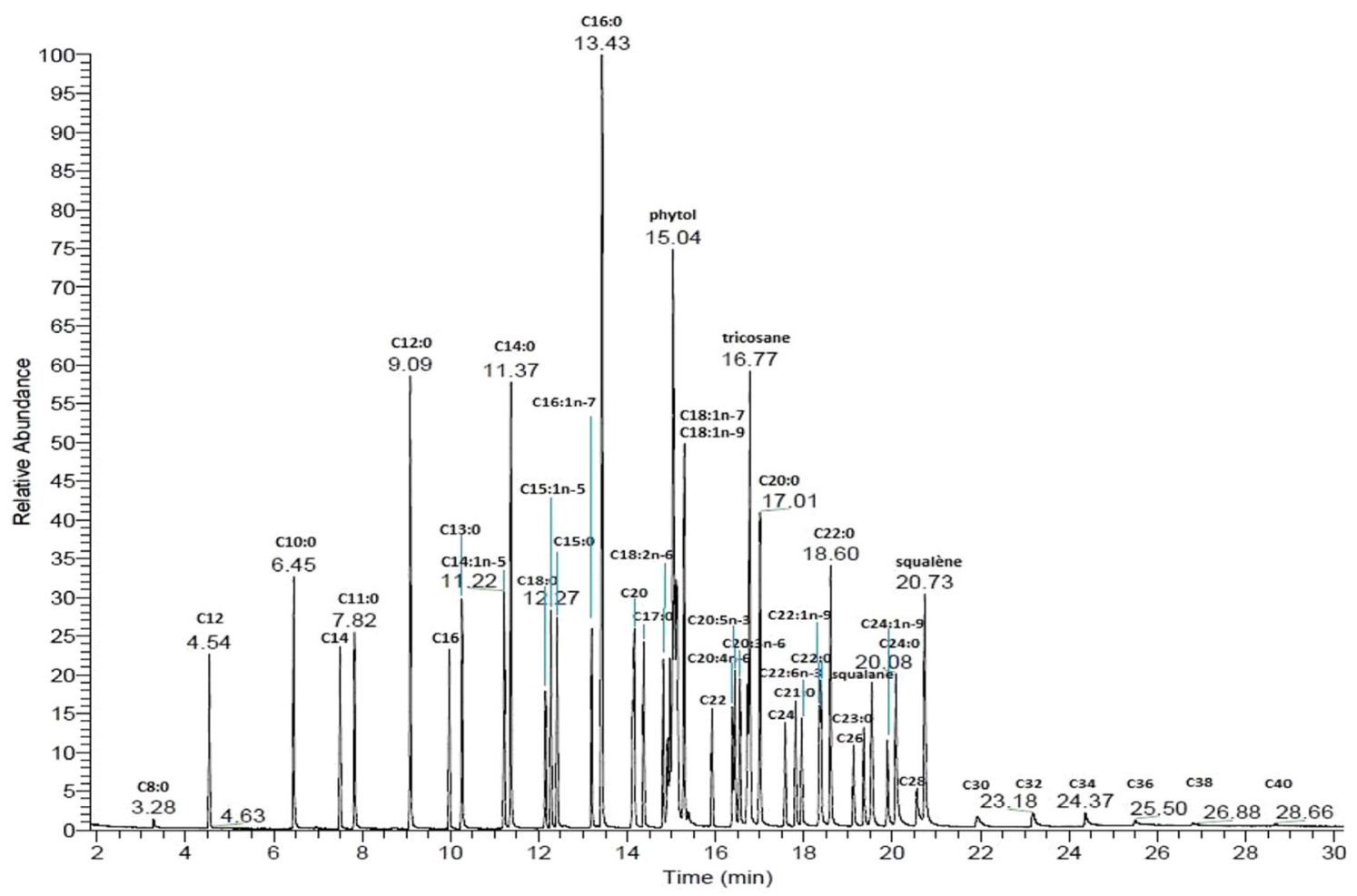

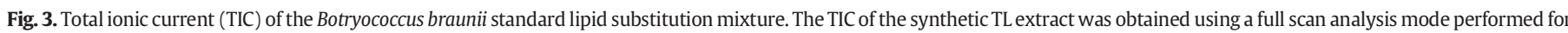
20 ng of injected compounds (each). 
discriminated between the FAME and hydrocarbon classes as illustrated on the factor map in Fig. S1. It was also possible to extract from this analysis a clear $m / z$ signature to explain the separation of the two groups of molecules. The $m / z$ choice was made according to the statistical parameter values calculated when performing the HCPC analysis. The parameters taken into account included the $v$ test (characterizing the contribution of the corresponding variable to the formed group), the $p$ value score (characterizing the significance of the result) and the overall mean relative intensity of the fragment ion considered (characterizing the sensitivity potential of the fragment ion, and taken into account as a priority) (Table S2). Thus the HCs were found to be represented by the fragments with $m / z 71,57,85$ and 43 , and the FAMEs by those with $m / z 74,55,41$ and 87 . These two signatures were in perfect agreement with the most commonly found ions in Silverstein (2005), confirming the pertinence of the analysis approach we selected. However, an exception occurred for squalene and the botryals, which were found to be clustered with compounds presenting a high degree of unsaturation. This indicated a prevalence of this chemical property and the need to address it thoroughly for the identification of the fragment ions permitting an optimized discrimination of the molecules studied.

A similar strategy was used to analyze each compound class independently, i.e. HCs or FAMEs separately, in order to look for discriminating factors between subgroups of molecules, and so identify consistent chemical subclasses. HCPC clustering results suggested the formation of five subgroups of molecules for the HCs and four subgroups for the FAMEs. Each subgroup could be correlated to common chemical properties of the molecules studied, thus pointing to distinct chemical subclasses (Fig. 4).

For the groups of HCs, the identification maps distinguished the saturated HCs with short carbon chains $C_{8}-C_{20}$, represented by the fragments with $m / z 29$ and 56 , and long carbon chains $C_{21}-C_{54}$, including squalane, represented by those with $m / z 113$ and 99 , from three subgroups of compounds specific to the Botryococcus genus, namely lycopadiene represented by the fragments with $m / z 125$ and 292, terpenes by those with $m / z 121$ and 136, and botryals by those with $m / z$

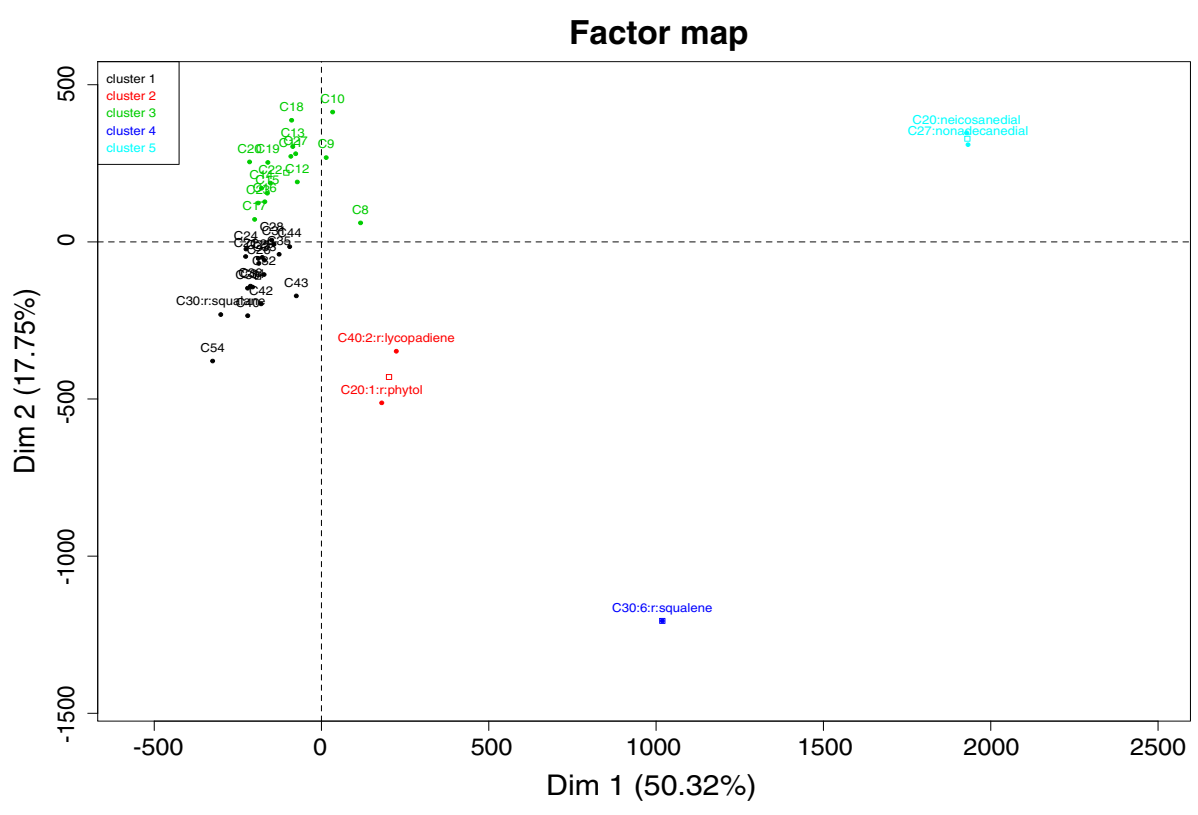

Factor map

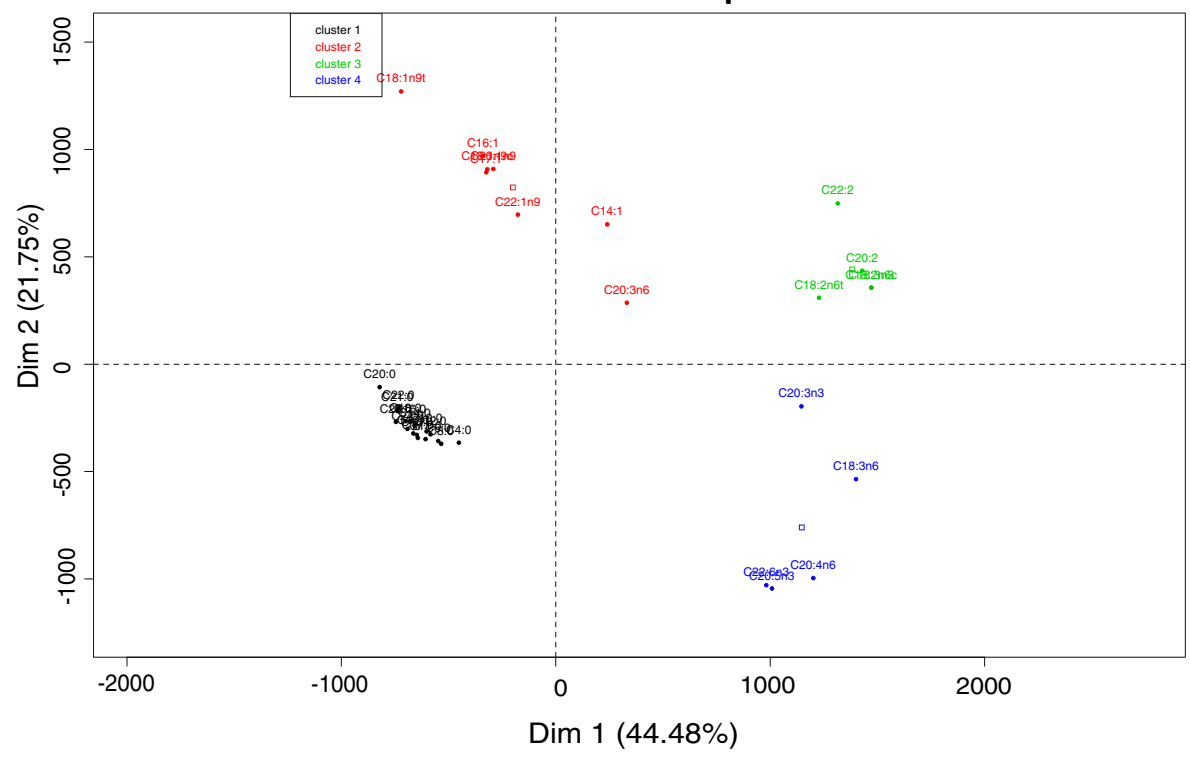

Fig. 4. HCPC clustering results for the HCs (top panel) and the FAMEs (bottom panel) according to their $m / z$ signatures. 
Table 4

Summary of the fragment ions selected as representative of the clusters of molecules obtained with the HCPC analysis.

\begin{tabular}{lll}
\hline $\begin{array}{l}\text { Chemical } \\
\text { class }\end{array}$ & Chemical subclass & Fragment ions $(m / z)$ \\
\hline HC & Saturated short-chain & \\
& Saturated long-chain (incl. squalane) & $71,57,85,43,29,56$ \\
& Mono- and di-unsaturated & $71,57,85,43,125,292$ \\
& (incl. lycopadiene) & \\
& Terpenes (incl. squalene) & $71,57,85,43,121,136$ \\
& Botryals & $71,57,85,43,82,95$ \\
FAME & Saturated & $74,55,41,87,143,75$ \\
& Mono-unsaturated & $74,55,41,87,69,83,97$ \\
& Di- and tri-unsaturated & $74,55,41,87,81,82,67$ \\
& Polyunsaturated & $74,55,41,87,79,91,80,93$ \\
\hline
\end{tabular}

82 and 95 . The specific case of squalene (and also terpenes) and botryals was also addressed when the compounds were mixed with all the FAMEs. HCPC clustering results found strictly identical complementary fragment ions for their discrimination, thus highlighting the robustness of the analysis protocol: terpenes with $\mathrm{m} / \mathrm{z} 121$ and 136 and botryals with $m / z 82$ and 95 .

For the FAMEs, subgroups of compounds were found according to their degree of unsaturation. One cluster was associated with the saturated fatty acids with $m / z 143$ and 75, others to the mono-unsaturated FAs with $m / z 55,69,83$ and 97 , to the di- or tri-unsaturated FAs with $m / z 81,82$ and 67 , and to the polyunsaturated FAs with $m / z 79,91,80$ and 93.

The properties of the chemical subclasses identified are summarized in Table S3, which gives the fragment ions best characterizing the HCs and FAMEs, Table 4, which gives the selected fragment ion signature (from S3 data) for each subclass of molecules, and Table S4, which shows their putative structures.

Table 5

Examples of S/N measured for some FAMEs and HCs (20 ng each) for five replicate chemotypes acquired in two detection modes: full scan (FS) and selected ion monitoring (SIM). ND: not detected. FC: fold change.

\begin{tabular}{|c|c|c|c|c|}
\hline Compound & FS mode $(\mathrm{S} / \mathrm{N})$ & SIM mode (S/N) & & $\mathbf{F C}(\mathbf{S} / \mathbf{N})$ \\
\hline Caprylic AME - 8:0 & 3543 & 6934 & I & 2 \\
\hline Dodecane - C12 & 79832 & 178391 & i & 2 \\
\hline Capric AME - C10:0 & 88671 & 181999 & i & 2 \\
\hline Tetradecane - C 14 & 82346 & 174678 & i & 2 \\
\hline Undecanoic AME - C11:0 & 65484 & 126416 & i & 2 \\
\hline Lauric AME - C12:0 & 158233 & 280359 & i & 2 \\
\hline Hexadecane - C16 & 85393 & 154707 & i & 2 \\
\hline Tridecanoic AME - C13:0 & 90857 & 148929 & i & 2 \\
\hline Myristic AME - C14:0 & 170239 & 294937 & i & 2 \\
\hline Octadecane- $\mathrm{Cl} 18$ & 68408 & 114550 & i & 2 \\
\hline Pentadecanoic AME - C15:0 & 88624 & 137176 & i & 2 \\
\hline Palmitic AME - C16:0 & 298346 & 459371 & i & 2 \\
\hline Icosane - C20 & 89966 & 140256 & i & 2 \\
\hline Heptadecanoic AME - C17:0 & 115 & 350 & i & 3 \\
\hline$\gamma$-linolenic AME - C 18:3n6 & 73996 & 46412 & i & 1 \\
\hline Linoleic AME - C18:2n6c & 50 & 96 & 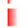 & 2 \\
\hline phytol & 514 & 600 & i & 1 \\
\hline Oleic AME - C 18: $\ln 9 c$ & 162 & 368 & I & 2 \\
\hline Stearic AME - C18:0 & 157711 & 222319 & i & 1 \\
\hline Docosane - C22 & 57497 & 89938 & i & 2 \\
\hline Arachidonic AME - C20:4n6 & 11 & 8 & i & 1 \\
\hline cis-5,8,11,14,17-eicosapentaenoic AME - C20:5n3 & 12 & 7 & i & 1 \\
\hline cis-8,11,14-eicosatrienoic AME - C20:3n6 & 33 & 139 & 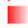 & 4 \\
\hline Tricosane - $\mathrm{C} 23$ & 40 & 124 & E & 3 \\
\hline Arachidic AME - C20:0 & 131841 & 173695 & I & 1 \\
\hline Tetracosane - C24 & 45162 & 79325 & i & 2 \\
\hline Heneicosanoic AME - C21:0 & 50742 & 86305 & I & 2 \\
\hline Behenic AME - 22:0 & 98047 & 235371 & i & 2 \\
\hline Hexacosane-C26 & 26769 & 100552 & E & 4 \\
\hline Tricosanoic AME - C23:0 & 37 & 44 & I & 1 \\
\hline Squalane - C30:r & 50957 & 183650 & E & 4 \\
\hline Nervonic AME - C24:1n 9 & 23 & 112 & E & 5 \\
\hline Lignoceric AME - C24:0 & 42140 & 181175 & 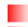 & 4 \\
\hline Octacosane - C28 & 23 & 82 & E & 4 \\
\hline Squalene - C30:6:r & 70361 & 207424 & E & 3 \\
\hline Triacontane - C30 & 1663 & 31921 & $=$ & 19 \\
\hline Dotriacontane - C32 & 1556 & 37764 & & 24 \\
\hline Tetratriacontane - C34 & 1298 & 37525 & & 29 \\
\hline Hexatriacontane - C36 & 561 & 15667 & & 28 \\
\hline Octatriacontane - C38 & 208 & 4625 & E & 22 \\
\hline Tetracontane - C40 & 53 & 1228 & & 23 \\
\hline
\end{tabular}

3.5. The selected $m / z$ signatures were used to develop a sensitive chemotyping method and assessed on substitution standard mixture for B. braunii and crude TL extracts

The fragment ions selected to characterize each chemical class or chemical subclass of the $B$. braunii targeted molecules enabled us to develop a specific GC-MS chemotyping method. This consisted in recording the MS data with a SIM mode programmed with the ion signature obtained in Section 3.4 (Table 3). The chemotyping method was assessed on the substitution standard mixture to evaluate its performance. For the same sample, the MS data acquired in SIM mode were compared with the MS data recorded in full scan mode under identical experimental separative conditions. Five independent TIC replicates were obtained and analyzed. The experimental variability was calculated from the area measurements, and the $\mathrm{S} / \mathrm{N}$ data extracted from each peak of interest. The results summarized in Table 5 were unambiguous. First, the calculated repeatability was judged acceptable, since the mean RSD calculated for every peak area was systematically less than $6 \%$. Second, a significant increase in the signal-to-noise ratio $(\mathrm{S} / \mathrm{N})$ was found especially for the HCs with high numbers of carbon atoms. For molecules exceeding 30 carbon atoms the gain in the $\mathrm{S} / \mathrm{N}$ ratio was calculated to be at least greater than $c a$. 20. The chemotyping method was also assessed on crude TL extract from the 10 strains of $B$. braunii studied grown in identical conditions for five independent replicates each. Results were also very clear-cut, similar repeatability (RSD ca. 8\%) and increase in $\mathrm{S} / \mathrm{N}$ ratio ( $\mathrm{ca} .20$ for the long-chain molecules) being found for the targeted molecules (data not shown).

Taken together, all these results indicate that the chemotyping method developed with MS data recorded in SIM mode was optimal for both sensitivity and specificity. It also argues for preferentially using SIM mode for sensitive chemotyping purposes, since it should maximize the chances of obtaining faithful chemical profiles of B. braunii TL crude extracts.

3.6. Application of the sensitive chemotyping approach for the rapid assessment of the biofuel potential of diverse B. braunii strains

Ten strains were tested in this study in order to assess our sensitive chemotyping approach: seven $B$. braunii strains belonging to race $A$ (bb1, bb2, bb4, bb7, bb8, bb9, bb10) and classified as producers of linear alkenes (di- or trienes), one strain of race B (bb3) producing mainly botryococcenes, i.e. triterpenes, and two strains (bb5 and bb6) of uncertain chemotaxon. All the strains were grown under similar conditions to assess minimal biomass concentration at an early stage of the culture, i.e. $0.5 \mathrm{~g} \cdot \mathrm{L}^{-1}$. The bb3 strain was also grown in totally $\mathrm{N}$-depleted conditions to see whether it could stimulate the biosynthesis of molecules of biofuel interest, i.e. botryococcenes (coded bb3bis). TL extraction was identical for the diverse biomasses tested, and our systematic rapid chemical profiling method applied. It was first performed in exploratory mode for the detection of as many non-targeted compounds as possible, i.e. in FS mode. On the same sample, the SIM mode was then used to enhance the detection and confirmation of the targeted lipid classes of interest. Data were manually processed and curated, allowing the detection of 80 different molecules for 11 conditions repeated five times each, yielding an $80 \times 55$ data matrix.

No qualitative difference was found when comparing the data matrices from either FS or SIM GCMS mode. As expected, the main difference was observed for the targeted lipids of interest when comparing the semi-quantitative data for alkene or botryococcene classes (data not shown). The matrix from the FS-GCMS chemotyping approach was therefore preferentially analyzed with the chemometric approach, since semi-quantification of the non-targeted molecules was not modified by the SIM programming. Chemotyping results were analyzed with the PCA approach, which highlighted clear differences between the strains, races and conditions tested. In Fig. 5 the factor maps indicate different behaviors in terms of averaged chemical profiles. The variables 
factor map allows an assessment of the chemotype associated with the strains tested, while the individuals factor map identifies the main compounds responsible for the observed differences. Strains bb2, 9, 7 and 8 were found to be clustered together as producers of alkadienes and alkatrienes of length at least $\mathrm{ca} .20$ equivalent-carbons (named $\mathrm{C} 20(2=)$ and $\mathrm{C} 22(3=)$ on principal component 1$)$. The recorded chemotypes confirmed here that these strains do belong to race A. As expected, the strain bb3 exhibited a chemotype totally orthogonal (principal component 2) to that of those measured for the B. braunii strains belonging to race $A$. This was mainly explained by the detection
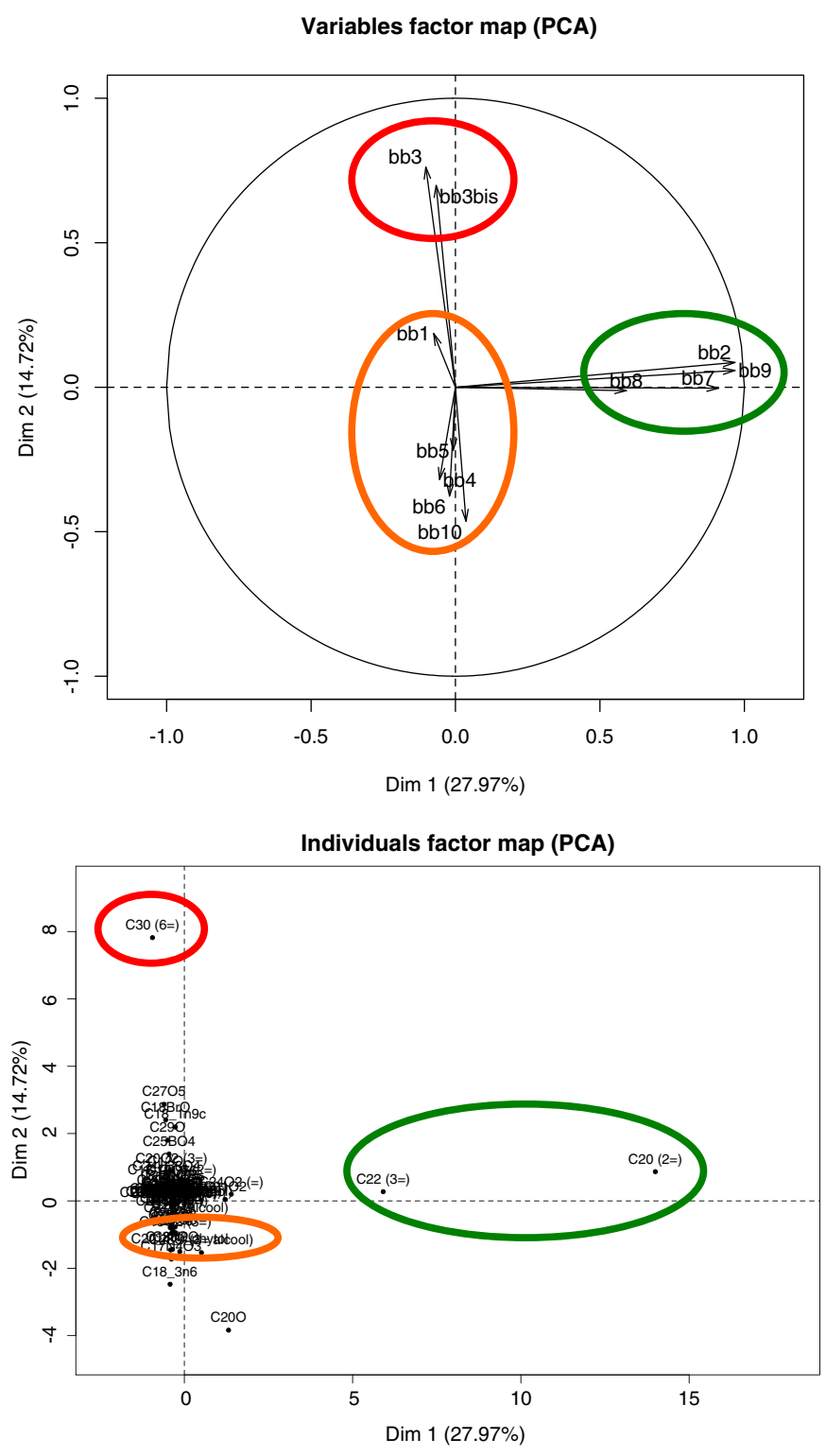

Fig. 5. PCA clustering results for the 10 strains of Botryococcus braunii profiled using the fast chemotyping method. The variables map at the top illustrates the projection of the tested $B$. braunii strains according to their chemical signature. The race B cluster is circled in red, the race A cluster in green and the other cluster in orange. The individuals map at the bottom illustrates the projection of the detected molecules for all the chemotypes, indicating their contribution for a given principal component. The molecules contributing the most are circled in red for race $\mathrm{B}$ (compound with chemical motif of at least 30 carbon atoms with 6 double bonds: $\mathrm{C} 30(6=))$, in green for race $\mathrm{A}$ (compounds with chemical motif of at least 20 carbon atoms with 2 or 3 double bonds: $\mathrm{C} 20(2=)$ and $\mathrm{C} 22(3=))$ and the other in orange (compound with phytyl as chemical motif). (For interpretation of the references to color in this figure legend, the reader is referred to the web version of this article.) of a major compound presenting the $m / z$ signature typical of the botryococcene producers of race B. Here the detected compound was of length ca. 30 equivalent-carbons, with at least 6 double bonds as found for the main structural motif of the botryococcenes (named C30 $(6=))$. The chemotype of the bb3bis strain, grown under Ndepleted condition, was very close to that of the bb3, grown under normal conditions, and exhibited no major difference for the molecules of interest, i.e. the botryococcenes. Another cluster grouping the bb1, bb5, bb4, bb6 and bb10 strains was found to be badly projected on the map. It corresponded to strains exhibiting very slow growth rate in our experimental conditions, with only pigments detected as major components, e.g. containing phytyl motifs. No compound characteristic of races A or B could be detected for those strains using our fast chemotyping strategy, suggesting that the physiological conditions used for the cultures were not well-adapted to the biosynthesis of botryococcenes, alkadienes or trienes. The chemotaxon of bb5 and 6 could not be determined here since these strains did not grow in the standard conditions tested. Further investigations will be needed first to improve their growing conditions and second to characterize their chemotype.

\section{Conclusion}

In this work we focused on developing a sensitive, rapid qualitative chemotyping approach based on chemometrically-assisted gas chromatography-mass spectrometry for the direct analysis of the crude TL extract of various $B$. braunii strains. We demonstrate that by using chemometrically selected fragment ions from low resolution MS spectra, it was first possible to identify chemical motifs characteristic of the lipids synthesized by our studied strain (namely alkenes and botryococcenes), without the standards. Second, these specific ion signatures were used to develop an original SIM-GCMS method used on both substitution standard solutions and real crude TL extracts from $10 \mathrm{~B}$. braunii strains. The experimental results show that the chemotyping approach was adequately sensitive, an $\mathrm{S} / \mathrm{N}$ enhancement of at least $\mathrm{ca}$. 20-fold being found for the molecules of interest. Our results also demonstrate the robustness of the combined use of the FS and SIM-GCMS method developed in real conditions, which was selective enough to unambiguously distinguish races of $B$. braunii strains, and in identical strains (or races) to differentiate cultural conditions and therefore physiological states.

Our proof of concept opens the way to a systematic, fast, convenient chemotyping of crude TL extracts obtained from $B$. braunii strains. The sensitivity and specificity of the method make it suitable for very small volume of biomass ( $5 \mathrm{~mL}$ of $0.5 \mathrm{~g} \cdot \mathrm{L}^{-1}$ concentrated biomass) and so support its use in systematic screening studies for the optimization of culture conditions and the influence of the environment on the lipid metabolism of $B$. braunii.

Supplementary data to this article can be found online at http://dx. doi.org/10.1016/j.algal.2015.05.001.

\section{Authors' contributions}

$\mathrm{BM}, \mathrm{MA}$ and DK contributed to the experimental section. DG, JP and JL contributed to the final content of the paper. OG supervised the work and drafted the manuscript.

\section{Acknowledgments}

Part of this work was funded by the CAER project (alternative fuel for aeronautics) and AIRBUS GROUP (Benjamin Moutel CIFRE Grant).

Special thanks are owed to Béatrice Legeais for careful reading and language editing of our manuscript. 


\section{References}

[1] A. Banerjee, R. Sharma, Y. Chisti, U.C. Banerjee, Botryococcus braunii: a renewable source of hydrocarbons and other chemicals, Crit. Rev. Biotechnol. 22 (3) (2002) 245-279.

[2] D.K. Barupal, T. Kind, S.L. Kothari, Y. Lee do, O. Fiehn, Hydrocarbon phenotyping of algal species using pyrolysis-gas chromatography mass spectrometry, BMC Biotechnol. 10 (2010) 40.

[3] E.G. Bligh, W.J. Dyer, A rapid method of total lipid extraction and purification, Can. J. Biochem. Physiol. 37 (8) (1959) 911-917.

[4] C. Chirac, E. Casadevall, C. Largeau, P. Metzger, Bacterial influence upon growth and hydrocarbon production of the green alga Botryococcus braunii1, J. Phycol. 21 (3) (1985) 380-387.

[5] V. Ebenezer, L.K. Medlin, J.S. Ki, Molecular detection, quantification, and diversity evaluation of microalgae, Mar. Biotechnol. (N.Y.) 14 (2) (2012) 129-142.

[6] J. Folch, M. Less, G.H. Sloane, A simple method for the isolation and purification of total lipids from animal tissues, J. Biol. Chem. 226 (1952) 497-509.

[7] J. Frenz, C. Largeau, E. Casadevall, F. Kollerup, A.J. Daugulis, Hydrocarbon recovery and biocompatibility of solvents for extraction from cultures of Botryococcus braunii, Biotechnol. Bioeng. 34 (6) (1989) 755-762.

[8] F. Gelin, J.W. de Leeuw, J.S. Sinninghe Dam, S. Derenne, C. Largeau, P. Metzger, Scope and limitations of flash pyrolysis-gas chromatography/mass spectrometry as revealed by the thermal behaviour of high-molecular-weight lipids derived from the green microalga Botryococcus braunii, J. Anal. Appl. Pyrolysis 28 (1994) 183-204.

[9] P.K. Kanaujia, Y.K. Sharma, M.O. Garg, D. Tripathi, R. Singh, Review of analytical strategies in the production and upgrading of bio-oils derived from lignocellulosic biomass, J. Anal. Appl. Pyrolysis 105 (2014) 55-74.

[10] E. Kojima, K. Zhang, Growth and hydrocarbon production of microalga Botryococcus braunii in bubble column photobioreactors, J. Biosci. Bioeng. 87 (6) (1999) 811-815.

[11] C. Largeau, E. Casadevall, C. Berkaloff, P. Dhamelincourt, Sites of accumulation and composition of hydrocarbons in Botryococcus braunii, Phytochemistry 19 (6) (1980) 1043-1051.

[12] S. Lê, J. Josse, F. Husson, FactoMineR: an R package for multivariate analysis, J. Stat. Softw. 25 (1) (2008) 1-18

[13] S. Meier, S.A. Mjos, H. Joensen, O. Grahl-Nielsen, Validation of a one-step extraction/ methylation method for determination of fatty acids and cholesterol in marine tissues, J. Chromatogr. A 1104 (1-2) (2006) 291-298.
14] P. Metzger, B. Allard, E. Casadevall, C. Berkaloff, A. Couté, Structure and chemistry of a new chemical race of Botryococcus braunii (Chlorophyceae) that produces lycopadiene, a tetraterpenoid hydrocarbon1, J. Phycol. 26 (2) (1990) 258-266.

[15] P. Metzger, A. Aumelas, Lycopanerols A, di-tetraterpenoid tetraether derivatives from the green microalga Botryococcus braunii, L strain, Tetrahedron Lett. 38 (17) (1997) 2977-2980.

[16] P. Metzger, E. Casadevall, Lycopadiene, a tetraterpenoid hydrocarbon from new strains of the green alga Botryococcus braunii, Tetrahedron Lett. 28 (34) (1987) 3931-3934.

[17] P. Metzger, E. Casadevall, M.J. Pouet, Y. Pouet, Structures of some botryococcenes: branched hydrocarbons from the B-race of the green alga Botryococcus braunii, Phytochemistry 24 (12) (1985) 2995-3002.

[18] P. Metzger, Y. Pouet, R. Summons, Chemotaxonomic evidence for the similarity between Botryococcus braunii L race and Botryococcus neglectus, Phytochemistry 44 (6) (1997) 1071-1075.

[19] P. Metzger, M.N. Rager, N. Sellier, C. Largeau, Lycopanerols I-L, four new tetraterpenoid ethers from Botryococcus braunii, J. Nat. Prod. 66 (6) (2003) 772-778.

[20] P. Metzger, E. Casadevall, Botryococcoid Ethers, Ether Lipids From Botryococcus braunii, Elsevier, Amsterdam, Netherlands, 1991.

[21] P. Metzger, M.N. Rager, Lycopanerols H, Two High Molecular Weight Ether Lipids From Botryococcus braunii Comprising An $\alpha$-tocopherol Unit, Elsevier, Kidlington, United Kingdom, 2002.

[22] S.A. Mjøs, The prediction of fatty acid structure from selected ions in electron impact mass spectra of fatty acid methyl esters, Eur. J. Lipid Sci. Technol. 106 (8) (2004) 550-560.

[23] M.N. Rager, P. Metzger, Six novel tetraterpenoid ethers, lycopanerols B-G, and some other constituents from the green microalga Botryococcus braunii, Phytochemistry 54 (4) (2000) 427-437.

[24] R. Razeghifard, Algal biofuels, Photosynth. Res. 117 (1-3) (2013) 207-219.

[25] R.M. Silverstein, F.X. Webster, D. Kiemle, Spectrometric Identification of Organic Compounds, 7th edition Wiley, 2005

[26] R. Suzuki, N. Ito, Y. Uno, I. Nishii, S. Kagiwada, S. Okada, T. Noguchi, Transformation of lipid bodies related to hydrocarbon accumulation in a green alga, Botryococcus braunii (race B), PLoS One 8 (12) (2013) e81626. 